\title{
Charged Higgs boson production beyond minimal flavor violation in the MSSM
}

\section{Michael Spannowsky ${ }^{* \dagger}$}

ITP, University of Karlsruhe, 76128 Karlsruhe, Germany

E-mail: mspeparticle.uni-karlsruhe.de

\begin{abstract}
In the Minimal Supersymmetric Standard Model squark mixing can significantly change the pattern of charged-Higgs production. We find that non-minimal flavor structures can have a sizeable impact on the charged-Higgs production cross section, whereas charged-Higgs searches may be able to probe flavor structures not accessible to rare kaon, bottom or charm experiments, and can invalidate the assumption of minimal flavor violation
\end{abstract}

Prospects for Charged Higgs Discovery at Colliders

September 16-19 2008

Uppsala, Sweden

\footnotetext{
*Speaker.

${ }^{\dagger}$ The author thanks Sven Heinemeyer for presenting the following material at the workshop.
} 


\section{Introduction}

While the light neutral Higgs particle is known to be the 'holy grail of particle physics', the charged Higgs particle can be rightly considered to be the same for an extended Higgs sector. To positively identify an extended Higgs sector, e.g. 2HDM or MSSM, it is not sufficient to study a light neutral Higgs alone [1]. However, the detection of a heavy charged Higgs is an unquestionable signal for new physics. Because of the absence of a $H^{ \pm} W^{\mp} Z$ vertex at tree level, the production of a single charged Higgs and its decay operate foremost via Yukawa couplings, whereas the heavy-quark Yukawa couplings to the heavy Higgs states are governed by $y_{b} \tan \beta$ and by $y_{t} / \tan \beta$. Unfortunately, for $2 \leq \tan \beta \leq 20$ the rates are small and no promising detection channel is known [2].

Within the Standard Model flavor symmetry breaking is governed solely by the Yukawa interactions, the spurions of flavor symmetry breaking. Applying this concept to extensions of the Standard Model leads to the notion of minimal flavor violation (MFV) [阿: in an MFV model there are no other sources of flavor violation than the Yukawa couplings. However, general soft SUSY breaking introduces new sources of flavor violation. In MFV (i) all soft scalar squark masses need to be diagonal in flavor space and (ii) all tri-scalar $A$-terms describing the squark-squark-Higgs couplings have to be proportional to the Yukawas. The concept of non-minimal flavor violation negates the assumptions (i) and (ii), inducing interactions between different squark generations.

$$
\begin{aligned}
\mathscr{L}_{\text {soft }}= & -\tilde{U}^{*} m_{\tilde{U}}^{2} \tilde{U}-\tilde{D}^{*} m_{\tilde{D}}^{2} \tilde{D}-\tilde{Q}^{\dagger} m_{\tilde{Q}}^{2} \tilde{Q} \\
& -\left[\tilde{Q} \bar{A}^{u} \tilde{U}^{*} H_{u}-\tilde{Q} \bar{A}^{d} \tilde{D}^{*} H_{d}+\text { h.c. }\right]
\end{aligned}
$$

The hermitian $6 \times 6$ squark mass matrices $\mathscr{M}_{q}^{2}$ for up and down-type squarks collect $D, F$ and soft terms from the SUSY breaking Lagrangian Eq.11.1). They are composed out of the left and right-handed blocks $M_{q A B}^{2}$. Each block is a $3 \times 3$ matrix in generation space:

$$
\mathscr{M}_{q}^{2}=\left(\begin{array}{cc}
M_{q L L}^{2} & M_{q L R}^{2} \\
M_{q L R}^{2 \dagger} & M_{q R R}^{2}
\end{array}\right)(q=u, d ; A, B=L, R) .
$$

The explicit expressions for the $M_{q A B}^{2}$ are given in [ [ new-physics flavor violation, it is useful to define the dimensionless mass insertions $\delta_{A B, i j}^{q} \equiv \frac{M_{q A B i j}^{2}}{\tilde{m}^{2}}$ [5], 6]. The denominator is the geometric mean $\tilde{m}^{2}=m_{A i i} m_{B j j}$ of the squared scalar masses of $\tilde{q}_{A i}$ and $\tilde{q}_{B j}$. The off-diagonal entries of $\delta_{A B}^{q}$ are significant only in non-MFV models and can be complex, inducing $C P$ violation. We confine ourselves to real $\delta_{A B}^{q}$. Note that in our numerical calculations we diagonalize the squark mass matrices and do not employ a perturbative expansion in the $\delta_{A B}^{q}$, avoiding the calculation of the squark unitary transformations [5].

Especially flavor physics can strongly constrains the free parameters from the soft-breaking lagrangian, relevant for the enhancement of the charged-Higgs production. Flavor violation among down squarks is more severely constrained, because in $K$ and $B$ physics down-squark effects can be mediated by strongly interacting gluino loops, while up-squark effects are mediated by the weak interaction. Furthermore, mixing between first and second generation squarks is tightly constrained 


$$
\begin{array}{lll}
\tan \beta=7 & m_{A}=170 \mathrm{GeV} & \mu=-300 \mathrm{GeV} \\
m_{\tilde{q}_{A A} i i}=600 \mathrm{GeV} & M_{2}=700 \mathrm{GeV} & m_{\tilde{g}}=500 \mathrm{GeV} \\
A^{u, c}=0 & A^{d, s, b}=0 & A^{t}=1460 \mathrm{GeV}
\end{array}
$$

Table 1: Generic parameter point

by $K$-physics and by the recent measurements of $D^{0} \bar{D}^{0}$-mixing. Hence, we can limit our analysis to up-squark mixing between the first/second and third generation while neglecting down-squark mixing, as long as it is not required by $S U(2)$. Particularly constraining are the radiative decays $B \rightarrow X_{s} \gamma$ and $B \rightarrow \rho \gamma$, the semileptonic decays $B \rightarrow X_{s} \ell^{+} \ell^{-}$and $B \rightarrow \pi \ell^{+} \ell^{-}$, and the $B_{d, s}-\bar{B}_{d, s}$ mass differences. A detailed discussion of the specific impact of each of the implented flavor bound can be found in [ $\llbracket$ ]. We find that the corresponding mass-matrix entries $A_{3 i}^{u}$ and $m_{\tilde{U}_{R} i 3}^{2}$ are only very loosely bounded by flavor physics ${ }^{1}$. The reason is that they involve right-handed squarks $\tilde{u}_{R}$ and $\tilde{c}_{R}$; those enter FCNC processes with external down quarks only via higgsino vertices proportional to the small up and charm Yukawa. Hence, the $\delta_{L R 3 i}^{u}$ and $\delta_{R R i 3}^{u},(i=1,2)$ are currently the least constrained flavored SUSY couplings. In the following we investigate the potential impact of these relevant $\delta_{3 i}^{u}$ on charged-Higgs collider searches.

\section{Single-Charged-Higgs Production}

We start by considering single-charged-Higgs production at tree level from quark-antiquark scattering, $q \bar{q}^{\prime} \rightarrow H^{ \pm}$, at the LHC. The amplitude for single-Higgs production in the type-II twoHiggs-doublet model is proportional to the quark Yukawa, thus small unless third-generation quarks are involved [7]. This chiral suppression is generic and with proper assumptions survives radiative corrections. For $\tan \beta=7$ and a charged-Higgs mass of $m_{H^{ \pm}}=188 \mathrm{GeV}$ the $H^{+}$production cross section at the LHC in the 2HDM is $41.2 \mathrm{fb}$, using the $\overline{\mathrm{MS}}$ quark masses given in [П].

The irreducible background to our searches is single- $W$ production, $q \bar{q}^{\prime} \rightarrow W^{ \pm}$. The $W^{+}$ production cross section of $90 \cdot 10^{6} \mathrm{fb}$ will be a serious challenge to our $H^{+}$search in the twoHiggs-doublet model.

Not assuming MFV has serious impact on the production rate for $q \bar{q}^{\prime} \rightarrow H^{ \pm}$. Squark loops will weaken the CKM suppression at the charged-Higgs-bottom vertex through flavor mixing. The dominant one-loop corrections are due to gluino vertex and self-energy diagrams. Beyond MFV, the loop diagrams do not have to include a (Dirac) quark mass to ensure the chiral limit of the theory. Instead, we can for example combine a Majorana mass with a left-right mixing $\delta_{L R}$ among the squarks. This combination can lift the supersymmetric charged Higgs production rate above the two-Higgs-doublet model prediction, despite its loop suppression. The MSSM Lagrangian we define at the weak scale, so all parameters are evaluated at the scale of the charged Higgs mass.

Our starting parameter point which does not violate any current bound is given in Table $1 . m_{A}$ denotes the mass of the CP-odd Higgs leading to $m_{H^{+}}=188 \mathrm{GeV} . M_{2}$ is the SUSY-breaking wino mass. The diagonal soft-breaking entries in the squark mass matrices we choose universal. All parameters are given at a scale of order $m_{H^{+}}$. The large value of $A^{t}$ (corresponding to $\delta_{L R, 33}^{u}$ ) increases

\footnotetext{
${ }^{1}$ We strictly use the convention $A_{i j}=A_{L_{i} R_{j}} \neq A_{j i}$.
} 

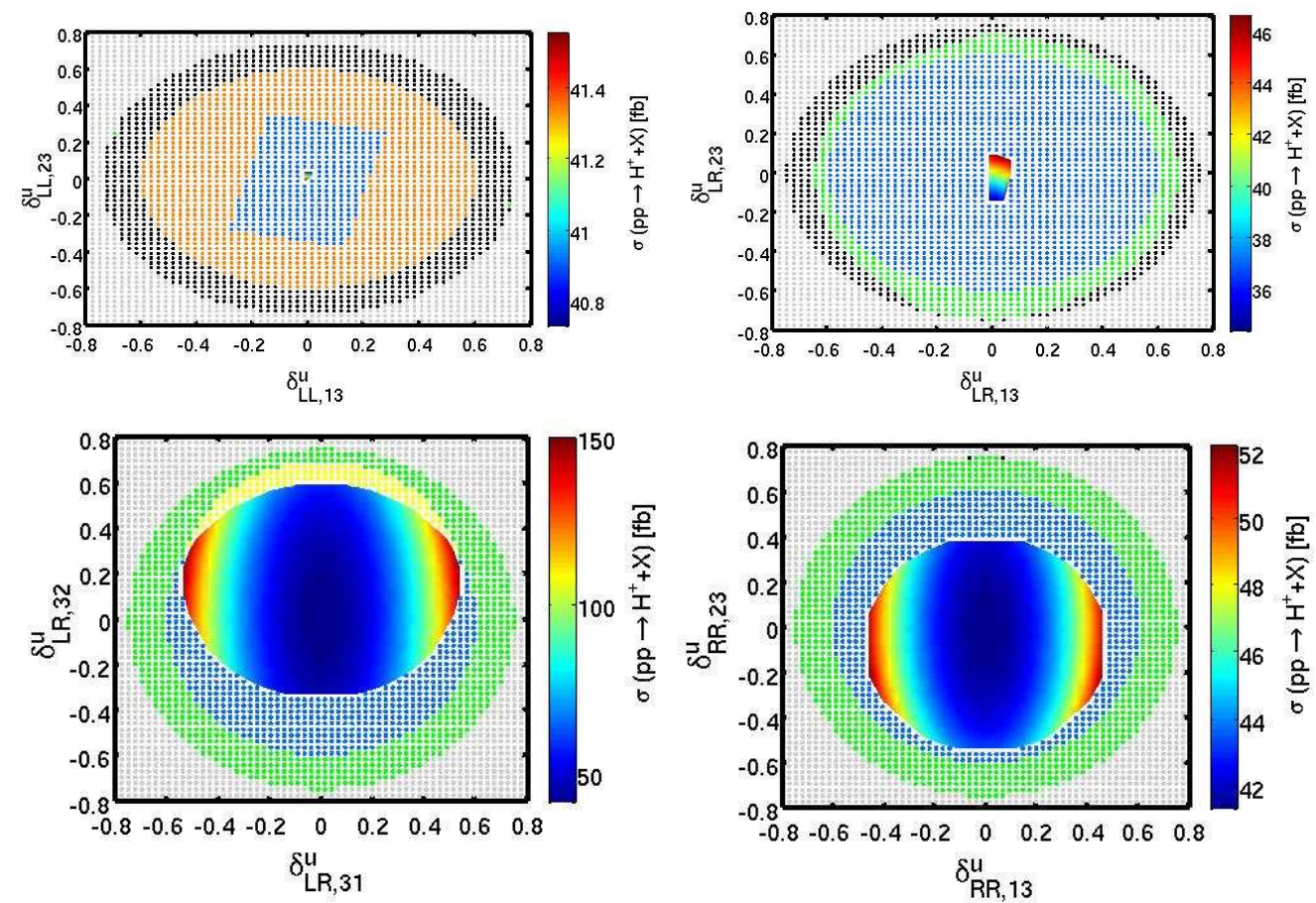

Figure 1: Single-charged-Higgs production rates at the LHC. In the rainbow-colored area we include beyond-MFV parameters around the parameter point Table 1. Two $\delta_{A B, i j}^{u}$ are varied in each panel, all others are set to zero. The area outside the rainbow is ruled out experimentally

the light Higgs mass to $119.9 \mathrm{GeV}$ at two loops. The production cross sections as a function of the three dominant beyond-MFV mass insertions in the up-sector are shown in Figure 1. Beyond-MFV effects can enhance the single-Higgs rate to values above $100 \mathrm{fb}$. The size of the production cross section is encoded in the rainbow scale in all panels of Fig. 1, while the parameter choices outside this area are ruled out. The different experimental constraints impacting the parameter point shown in Fig. 11 include:

- Tevatron searches rule out the yellow points.

- squark searches and radiative and semileptonic decay limits rule out the green points.

- black points are forbidden by the squark-mass limits, $B$ mixing, and radiative and semileptonic decays.

- blue points indicate a violation of the radiative and semileptonic decay bounds only.

- orange points correspond to a violation of the $B$ mixing and radiative and semileptonic decay limits.

- grey points on the outside of the panels indicate a negative squark mass square after diagonalizing the squark mass matrix. 


\begin{tabular}{|c|c|c|c|c|c|c|c|}
\hline$m_{H^{+}}$ & $\tan \beta$ & \multicolumn{2}{|c|}{$\sigma_{2 \mathrm{HDM}}$} & \multicolumn{2}{|c|}{$\sigma_{\mathrm{MFV}}$} & \multicolumn{2}{|c|}{$\sigma_{\mathrm{MFV}}^{\left(m_{f}=0\right)}$} \\
\hline $188 \mathrm{GeV}$ & 3 & \multicolumn{2}{|c|}{$2.5 \cdot 10^{-1}$} & \multicolumn{2}{|c|}{$2.6 \cdot 10^{-1}$} & \multicolumn{2}{|c|}{$6.7 \cdot 10^{-4}$} \\
\hline $188 \mathrm{GeV}$ & 7 & \multicolumn{2}{|c|}{$9.9 \cdot 10^{-1}$} & \multicolumn{2}{|c|}{$1.1 \cdot 10^{0}$} & \multicolumn{2}{|c|}{$1.5 \cdot 10^{-4}$} \\
\hline \multirow{2}{*}{$\begin{array}{l}400 \mathrm{GeV} \\
400 \mathrm{GeV}\end{array}$} & 3 & \multicolumn{2}{|c|}{$4.0 \cdot 10^{-2}$} & \multicolumn{2}{|c|}{$4.2 \cdot 10^{-2}$} & \multicolumn{2}{|c|}{$4.2 \cdot 10^{-4}$} \\
\hline & 7 & \multicolumn{2}{|c|}{$1.6 \cdot 10^{-1}$} & \multicolumn{2}{|c|}{$1.7 \cdot 10^{-1}$} & \multicolumn{2}{|c|}{$9.1 \cdot 10^{-5}$} \\
\hline \multicolumn{2}{|c|}{$m_{H^{+}}$} & $\tan \beta$ & $\sigma_{\mathrm{SUS}}$ & & $\sigma_{\mathrm{SU}}^{\left(m_{f}\right.}$ & $\sigma_{\text {SUSY }}$ & \\
\hline \multicolumn{2}{|c|}{$188 \mathrm{GeV}$} & 3 & \multicolumn{2}{|c|}{14.3 . } & \multicolumn{3}{|c|}{$13.9 \cdot 10^{0}$} \\
\hline \multicolumn{2}{|c|}{$188 \mathrm{GeV}$} & 7 & 4.6 . & & \multicolumn{3}{|c|}{$3.0 \cdot 10^{0}$} \\
\hline \multicolumn{2}{|c|}{$400 \mathrm{GeV}$} & 3 & & & \multicolumn{3}{|c|}{$2.3 \cdot 10^{0}$} \\
\hline \multicolumn{2}{|c|}{$400 \mathrm{GeV}$} & 7 & 7.9 . & $10^{-1}$ & \multicolumn{3}{|c|}{$5.4 \cdot 10^{-1}$} \\
\hline
\end{tabular}

Table 2: Production rates (in fb) for the associated production of a charged Higgs with a hard jet: $p_{T, j}>$ $100 \mathrm{GeV}$. The label $2 \mathrm{HDM}$ denotes a two-Higgs-doublet of type II, while MFV and SUSY refer to the complete set of supersymmetric diagrams, assuming MFV and beyond. Beyond MFV we choose $\delta_{L R, 31}^{u}=$ 0.5 .

In Fig. 1 we see that the limits on radiative and semileptonic decays followed by the Tevatron limit on light-flavor squark masses define two distinct boundaries of forbidden parameter space. After taking into account all limits, the off-diagonal entry $\delta_{L R, 31}^{u}$ has the strongest impact on the rate.

\section{Charged-Higgs Production with a hard Jet}

The generic chiral suppression that characterizes single-Higgs production and limits the cross section at tree level can be removed by simply adding an external gluon to the operator basis, leading to higher-dimensional $q \bar{q}^{\prime} H g$ operators after electroweak symmetry breaking [8]. To probe such operators at the LHC, we study charged-Higgs searches in association with a hard jet. Possible large supersymmetric corrections in this process can only occur beyond MFV - just like for single-Higgs production. Simple diagrams for this process can be derived from all single-Higgs production diagrams just radiating an additional gluon. These are infrared divergent, which is no problem once we require a hard jet with a typical $p_{T, j}>100 \mathrm{GeV}$. The dominant background to this signature is clearly $W+$ jet production $\sigma\left(p p \rightarrow W^{+}\right.$Jet $) \approx 1 \mathrm{nb}$, with the $W$ decaying to a hadronic $\tau$.

We know from single-Higgs production that the flavor effects we are interested in can be much larger than we expect next-to-leading order QCD effects to be. Therefore, we ignore all gluonic next-to-leading order corrections to charged-Higgs production with a hard jet and limit our analysis to tree-level rates in the two-Higgs-doublet model and additional supersymmetric one-loop corrections.

Assuming MFV, $F$-term and $A$-term couplings of the Higgs to two squarks are proportional to the quark masses, which means that supersymmetric one-loop amplitudes are expected to be of the size of typical supersymmetric NLO corrections. In the first column of Table 2 we list the 
hadronic tree level cross sections for charged Higgs plus jet production for a non-supersymmetric two-Higgs-doublet type-II model. Numerical results for hadronic charged Higgs plus jet production in MFV we present in the second column in Table 2 .

In the limit $m_{f} \rightarrow 0$ just the $D$-terms do contribute. Although chirally not suppressed and enhanced for small $\tan \beta$, the $D$-term contribution is only a small fraction of the supersymmetric amplitude, due to its faster decoupling with heavy superpartner masses $\sigma \propto 1 / M_{\mathrm{SUSY}}^{8}$.

Although the operator basis does not get significantly extended when we include beyond-MFV effects, the effective couplings will get enhanced once we allow for sizeable $\delta_{A B, i j}^{u}$. We respect the results form Section 2 , where we found $\delta_{R L, 13}^{u}$ to amplify the charged-Higgs cross section most. In the lower pattern of Tab. Z we see, that, independent of the Yukawa couplings, beyond MFV can enhance the rate in the region of small $\tan \beta$ significantly, compared to the tree-level or MFV process.

\section{Conclusion}

We find that if we allow for general squark mixing the cross sections for single-charged-Higgs production and charged-Higgs production in association with a hard jet can be enhanced by an order of magnitude, even after including all current experimental bounds.

The dominant source of genuine supersymmetric flavor enhancement in the charged-Higgs production rate is the soft-breaking $A$ term for up-type squarks $A_{i 3}^{u}$, which is invisible to kaon, charm and $B$-experiments. Hence, collider searches for enhanced charged-Higgs production rates can probe a unique sector of flavor. A discovery would besides a breakdown of the Standard Model also signal a non-standard solution to the flavor puzzle beyond the minimal-flavor-violation hypothesis. Unfortunately, at present, we cannot firmly claim that these flavor-induced chargedHiggs production rates at small $\tan \beta$ rates lead to observable signals over the large $W$-production backgrounds.

\section{References}

[1] M. Dührssen, S. Heinemeyer, H. Logan, D. Rainwater, G. Weiglein and D. Zeppenfeld, Phys. Rev. D 70, 113009 (2004)

[2] CMS Collaboration, Nucl. Part. Phys. 34, 995 (2007)

[3] G. D’Ambrosio, G. F. Giudice, G. Isidori and A. Strumia, Nucl. Phys. B 645, 155 (2002).

[4] S. Dittmaier, G. Hiller, T. Plehn and M. Spannowsky, Phys. Rev. D 77, 1150001 (2008).

[5] L. J. Hall, V. A. Kostelecky and S. Raby, Nucl. Phys. B 267, 415 (1986).

[6] J. S. Hagelin, S. Kelley and T. Tanaka, Nucl. Phys. B 415, 293 (1994); F. Gabbiani, E. Gabrielli, A. Masiero and L. Silvestrini, Nucl. Phys. B 477, 321 (1996);

[7] J. L. Diaz-Cruz, H. J. He and C. P. Yuan, Phys. Lett. B 530, 179 (2002); H. J. He and C. P. Yuan, Phys. Rev. Lett. 83, 28 (1999);

[8] C. N. Leung, S. T. Love and S. Rao, Z. Phys. C 31, 433 (1986); W. Buchmüller and D. Wyler, Nucl. Phys. B 268, 621 (1986). 